53 RUNNING A LEADERSHIP SOCIETY DURING THE COVID19 PANDEMIC - THE SWITCH TO A VIRTUAL PLATFORM

Prisca Singh, Shihabul Hassan, Haroon Ali Shah. Queen's Hospital, Burton, England

10.1136/leader-2021-FMLM.53

Aims Birmingham Medical Leadership Society (BMLS) is a society that aims to promote leadership within healthcare to students at the University's Medical and Dental School. We have worked to convert our usual events to an online format, so that we can continue to provide leadership-oriented events during the Covid-19 pandemic. This abstract highlights key considerations for conversion to an online platform, and shows the outcomes of BMLS' approach.

Methods The events were analysed according to results from the online feedback forms collected post-event. This data was analysed using Microsoft Excel 2016.

Results The feedback has shown that the majority of students are satisfied with our virtual events, with an average rating 4.63 out of 5 for the usefulness of the session, and 4.71 for how interesting it was. Additionally, the average score for relevance of the event to the viewer was 4.67. This demonstrates that online delivery of events can be effective at engaging medical students with leadership topics.

Conclusions Delivering leadership events during a pandemic has its challenges, but by switching to online platforms it's possible to engage an audience in numerous events. Both Zoom, a more traditional option, and also Instagram Live are valuable tools. When hosting an event on either platform, it's crucial to plan adequate promotion of the event beforehand, as well as getting all members of the committee comfortable with the platform that will be used and doing run through sessions to ensure all links are appropriate. Feedback forms should also be designed for each session. By preparing for a virtual event this way, societies can make a smooth transition to online event provision. In this way, leadership can continue to be promoted to medical students in the UK.

\section{SERVICE TRANSFORMATION OF ACUTE HOME VISITING SERVICE (AHVS) DURING COVID-19 PANDEMIC}

${ }^{1}$ Reshma Rasheed, 'Babafemi Salako, ' Olugbenga Odutola, ${ }^{2}$ Yathorshan Shanthakumaran. ${ }^{1} B B$ Healthcare Solutions, UK; ${ }^{2}$ University of Varna, Bulgaria

\subsection{6/leader-2021-FMLM.54}

Background The rapidly evolving situation of COVID-19 pandemic made it imperative for the existing home visiting service to transform. Within a period of days, protocols were developed, staff training was undertaken and adequate infection control measures were deployed.

Aims/Objectives Identify key aspects for the redeployment of Acute Home Visiting Service through COVID-19 pandemic

Methods A 4-week pilot exercise was initiated; we used quantitative and qualitative feedback from users to implement further changes. This enabled us to calculate the estimated demand of the service and additional staff that was required. We rapidly deployed COVID-19 policies, procedures and protocols as a result. We trained our staff to deliver the home visiting services working collaboratively with existing community and primary care services. All visits were triaged by senior clinicians to ensure a risk assessment of the visit in COVID19 pandemic conditions was conducted. An ongoing service evaluation was performed.
Results A total of 11,478 appointments were recorded, of which 9990 (87\%) were avoidable hospital admissions. We avoided hospital admissions at a time when services were already strained. A daily, and weekly workload and admissions information were provided to Basildon and Brentwood CCG.

Conclusions Primary care services underwent rapid transformation across all service lines, the Prime Minister's Challenge Fund primed GP federations that were able to work at scale within a short period of time and remained responsive to the primary care challenges of home visiting and admission avoidance to protect secondary care services.

\section{SIMULATION VIA INSTANT MESSAGING - BIRMINGHAM ADVANCE (SIMBA) EFFECTIVELY DEVELOPS LEADERSHIP AND INTERPERSONAL SKILLS IN MEDICAL STUDENTS AND JUNIOR DOCTORS}

${ }^{1}$ Rachel Nirmal*, ${ }^{1}$ Isabel Allison, ${ }^{2}$ Cai Ying Ng, ${ }^{2}$ Emma Ooi, Georgia Morgan, ${ }^{3}$ Meri Davitadze, ${ }^{4,5}$ Eka Melson, ${ }^{5,6}$ Punith Kempegowda. ' University of Birmingham Medical School, Birmingham, UK; ${ }^{2}$ RCSI and UCD Malaysia Campus, Penang, Malaysia; ${ }^{3}$ GeorgianAmerican Family Medicine Clinic 'Medical House', Tbilisi, Georgia; ${ }^{4}$ Ninewells Hospital, NHS Tayside, Dundee, UK; ${ }^{5}$ Institute of Metabolism and Systems Research, University of Birmingham Birmingham, UK; ${ }^{6}$ Department of Endocrinology, Queen Elizabeth Hospital, University Hospitals Birmingham NHS Foundation Trust, Birmingham, UK

\subsection{6/leader-2021-FMLM.55}

Background Simulation via Instant Messaging - Birmingham Advance (SIMBA) is a simulation-based learning model increasing clinicians' confidence in managing various clinical scenarios. The international team delivering SIMBA sessions is composed of medical students and junior doctors. Executing these sessions requires team members to lead peers, communicate with senior doctors, and delegate roles to ensure completion of case preparation, moderator training, and advertisement.

Objectives We studied whether SIMBA affects the leadership and teamwork skills of medical students and junior doctors.

Methods 33 medical students and junior doctors were included in the study based on their experience in leadership roles in SIMBA. Participants completed open-ended questions, Leadership Trait Questionnaire (LTQ) and Teamwork Skills Questionnaire (TSQ) twice; first as they would respond at present, and second as they would have responded prior to joining SIMBA. Pre- and post-SIMBA involvement scores were compared for each of the measured traits from both questionnaires and analysed using Wilcoxon signed-rank test. Common themes were identified and inductive thematic analysis was performed.

Results On average, each participant had spent six months in SIMBA. Post-SIMBA evaluation showed significant improvement in 9/14 LTQ traits $(\mathrm{p}<0.05)$, and all 6 TSQ traits $(\mathrm{p}<0.0001)$. The most notable improvements measured by the LTQ were in determined and persistent subscales, followed by dependable and diligent. The largest improvements observed by the TSQ were in decision-making, communication skills, and adaptability. Responses to open-ended questions reported positive effects on confidence, organisation, and interpersonal skills ( $n=28 / 33,84.8 \%)$.

Conclusions Leadership and teamwork skills are vital for medical students and junior doctors. Our study proves that involvement with a novel platform such as SIMBA is effective in fostering these skills at an early career stage. 